\title{
Holographic subregion complexity under a thermal quench in an Einstein-Maxwell-axion theory with momentum relaxation
}

\author{
Yu-Ting Zhou, ${ }^{1,2, \dagger}$ Xiao-Mei Kuang $\oplus^{2,4, *}$ Yong-Zhuang Li®, ${ }^{3, \ddagger}$ and Jian-Pin Wu ${ }^{2,4, \S}$ \\ ${ }^{1}$ College of Mathematics and Science, Yangzhou University, Yangzhou 225009, China \\ ${ }^{2}$ Center for Gravitation and Cosmology, College of Physical Science and Technology, \\ Yangzhou University, Yangzhou 225009, China \\ ${ }^{3}$ School of Science, Jiangsu University of Science and Technology, Zhenjiang 212003, China \\ ${ }^{4}$ School of Aeronautics and Astronautics, Shanghai Jiao Tong University, Shanghai 200240, China
}

(Received 14 December 2019; accepted 15 May 2020; published 26 May 2020)

\begin{abstract}
We investigate the evolution of holographic entanglement entropy (HEE) and holographic complexity (HC) under a thermal quench in Einstein-Maxwell-axion theory, which is dual to a field theory with momentum relaxation on the boundary. A strip-shaped boundary geometry is utilized to calculate HEE and $\mathrm{HC}$ via "entropy = surface" and "complexity = volume" conjecture, respectively. By fixing other parameters, we claim that either large enough black hole charge or width of the strip will introduce swallow-tail behaviors in HEE and multivalues in HC due to the discontinuity of the minimum HubenyRangamani-Takayanagi surface. Meanwhile, we explore the effects of momentum relaxation on the evolution of HEE and HC. The results present that the momentum relaxation will suppress the discontinuity to occur as it increases. For large enough momentum relaxation, the continuity of HEE and HC will be recovered.
\end{abstract}

DOI: $10.1103 /$ PhysRevD.101.106024

\section{INTRODUCTION}

Holography [1-3] has provided a close connection among the quantum information, condensed matter, and quantum gravity. These connections become more and more important in the realm of theoretical physics and quantum information. From the view of technics, it is also a powerful tool to study many physical quantities in these areas, especially in strongly correlated systems.

Among the physical quantities, entanglement entropy (EE) and complexity are two significant concepts in the theoretical physics and quantum information. Essentially, EE measures the degrees of freedom in a strongly coupled system, while the complexity measures the difficulty of turning a quantum state into another state. But it is extremely difficult to evaluate them on the side of the field theory when the degrees of freedom of the system become large. Fortunately, both of them can be evaluated with the help

\footnotetext{
*Corresponding author. xmeikuang@yzu.edu.cn

Published by the American Physical Society under the terms of the Creative Commons Attribution 4.0 International license. Further distribution of this work must maintain attribution to the author(s) and the published article's title, journal citation, and DOI. Funded by SCOAP ${ }^{3}$. of holography and their elegant geometric duality from gravity side has been provided. Specially, in the holographic framework, it has been proposed that in $[4,5]$ the EE for a subregion on the dual boundary is proportional to the minimal Hubeny-Rangamani-Takayanagi (HRT) surface in the bulk geometry. Later, two different methods were proposed to evaluate the complexity from geometry. One is the "complexity = volume" $(\mathrm{CV})$ conjecture, which states that the holographic complexity (HC) is proportional to the volume of a codimension-one hypersurface with the antide Sitter (AdS) boundary and the HRT surface [6,7]. While the other is "complexity = action" conjecture in which one identifies $\mathrm{HC}$ with the gravitational action evaluated on the Wheeler-DeWitt patch in the bulk $[8,9]$. Some analytical treatment in this setup has been addressed in $[10,11]$. In this paper, we will follow the CV conjecture and study its evolution under a thermal quench.

In the framework of holography, some of related quantities and their geometric descriptions have been holographically investigated. For instance, entanglement of purification (EoP), which is an important quantum information quantity for mixed states, has been holographically dual to the minimal entanglement wedge cross section [12-14] and generalized in [15-17]. The bit thread formalism for studying EoP was then addressed in [17-20]. Complexity of purification $(\mathrm{CoP})$, which describes the minimum number of gates needed to purify a mixed state, was holographically explored in $[17,21]$. Another interesting 
quantity is the logarithmic negativity which captures the quantum correlations with the nature of entanglement [22]. It is also a quantum entanglement measure for mixed quantum states and the holographic dual has been studied in [23,24].

Additionally, the study of $\mathrm{HEE}$ and $\mathrm{HC}$ will provide us more indirect but effective information to explore the nature of the spacetime, in particular the physics of the black hole horizon and its thermal and entanglement structures. Specifically, it was shown in $[25,26]$ that EE is not enough to understand the physics of the black hole horizon via studying the information paradox in black holes. Therefore, the authors proposed the ER = EPR conjecture [27] and argued that the creation of the fire wall behind the horizon is essentially a problem of quantum computational complexity [6,7], where ER and EPR stand for Einstein-Rosen bridges and Einstien-Podolsky-Rosen correlations, respectively. The evolution of the HEE and HC under a thermal quench has been explored in various dynamical backgrounds such as Einstein theory [28-30], Einstein-Born-Infeld theory [31], and massive gravity theory [32], and has been further generalized to chaotic system [33] and dS boundary [34]. The evolution of HEE and $\mathrm{HC}$ for quantum quench has also been investigated in [35-39] and references therein.

In this paper, we will investigate the evolution of HEE and $\mathrm{HC}$ under a thermal quench in Einstein-Maxwell gravity coupled with two linear spacial-dependent scalar fields in the bulk, which is called Einstein-Maxwell-axion (EMA) theory. The analytical black brane solution with dimension $D \geq 4$ was constructed in [40], and it was found that four-dimensional black brane geometry is that of a sector of massive gravity [41]. Moreover, the evolution of $\mathrm{HEE}$ and $\mathrm{HC}$ for different dimensions of Einstein theory has been studied in [28]. So here we shall focus on the simplest four-dimensional case in this setup which is dual to three-dimensional boundary theory. It was addressed in [40] that the scalar fields in the bulk source a spatially dependent field theory with momentum relaxation, while the linear coefficient of the scalar fields describes the strength of the momentum relaxation. This means that our study will involve in momentum relaxation, which is more closer to the reality. Moreover, the effect of momentum relaxation on the time evolution of the optical conductivity [42] and the equilibrium chiral magnetic effect [43] with Vaidya quench in this model have been studied, which shed light on the quark gluon plasma produced in heavy ion collisions as well as the real-world systems. It is worthwhile to point out that in this model, momentum relaxation in the dual boundary is sourced by the bulk scalar fields in the bulk, but the black hole geometry is homogeneous as we will present soon. The fully inhomogeneous holographic thermalization process with spacial-dependent bulk geometry has been studied in [44].

Our paper is organized as follows. In Sec. II, we study the generalized Vaidya-AdS black brane in EMA theory. Then, in Sec. III, we present the holographic setup of HEE and $\mathrm{HC}$ for a stripe geometry. We show our results and analyze the effect of momentum relaxation on the evolution of HEE and HC in Sec. IV. Finally, Sec. V contributes to our conclusions and discussions. In this paper, we will set the units as $G=\hbar=c=1$.

\section{VAIDYA ADS BLACK BRANES IN EINSTEIN- MAXWELL-AXION GRAVITY THEORY}

We consider the AdS black branes in EMA gravity proposed in [40]. The action of the four-dimensional theory is given by

$S=\frac{1}{16 \pi} \int d^{4} x \sqrt{-g}\left(R+\frac{6}{\ell^{2}}-\frac{1}{4} F_{\mu \nu} F^{\mu \nu}-\frac{1}{2} \sum_{I=1}^{2}\left(\partial \psi_{I}\right)^{2}\right)$.

By setting the scalar fields to linearly depend on the twodimensional spatial coordinates $x^{a}$, i.e., $\psi_{I}=\beta \delta_{I a} x^{a}$ where the index $a$ goes $a=1,2$, the action admits the charged black brane solution

$$
\begin{aligned}
d s^{2} & =-r^{2} f(r) d t^{2}+\frac{1}{r^{2} f(r)} d r^{2}+r^{2}\left(d x^{2}+d y^{2}\right), \\
A & =A_{t}(r) d t, \quad \text { with } \\
f(r) & =\frac{1}{\ell^{2}}-\frac{\beta^{2}}{2 r^{2}}-\frac{m}{r^{3}}+\frac{q^{2}}{r^{4}}, \\
A_{t} & =\left(1-\frac{r_{h}}{r}\right) \frac{2 q}{r_{h}} .
\end{aligned}
$$

Here, the horizon $r_{h}$ satisfies $f\left(r_{h}\right)=0 ; \ell$ describes the radius of AdS spacetime, and for simplicity, we will set $\ell=1 . m$ and $q$ are the mass and charge of the black brane, respectively, with the relation given by

$$
1-\frac{\beta^{2}}{2 r_{h}^{2}}-\frac{m}{r_{h}^{3}}+\frac{q^{2}}{r_{h}^{4}}=0 .
$$

It is worthwhile to point out that the scalar fields in the bulk source a spatially dependent boundary field theory with momentum relaxation, which is dual to a homogeneous and isotropic black brane (2). The linear coefficient $\beta$ of the scalar fields is usually considered to describe the strength of the momentum relaxation in the dual boundary theory [40]. A general action with axions terms and the holography has been studied in [45]. We note that the extended thermodynamics of the black brane has also been studied in [46-48]. The Hawking temperature and the thermal entropy density of the black brane read

$$
T=\left.\frac{1}{4 \pi} \frac{d\left(r^{2} f(r)\right)}{d r}\right|_{r_{h}}, \quad s=4 \pi r_{h}^{2},
$$


which is treated as the temperature of the dual boundary field.

With properly chosen coordinate transformation, the above black hole brane (2) can be represented as in the Eddington-Finkelstein coordinates,

$$
\begin{gathered}
d s^{2}=\frac{1}{z^{2}}\left[-f(z) d v^{2}-2 d v d z+d x^{2}+d y^{2}\right], \\
f(z)=1-\frac{1}{2} \beta^{2} z^{2}-m z^{3}+q^{2} z^{4}, \quad A_{v}=2 q(1-z),
\end{gathered}
$$

with

$$
d v=d t-\frac{1}{f(z)} d z \quad \text { and } \quad z=\frac{1}{r} .
$$

We note that the coordinates $v$ and $t$ coincide on the boundary. Thus, in order to holographically describe the evolution of HEE and HC, one usually frees the mass and charge parameter as smooth functions of $v$ as $[49,50]$

$$
\begin{aligned}
& M(v)=\frac{m}{2}\left[1+\tanh \left(\frac{v}{v_{0}}\right)\right], \\
& Q(v)=\frac{q}{2}\left[1+\tanh \left(\frac{v}{v_{0}}\right)\right],
\end{aligned}
$$

where $v_{0}$ represents the finite thickness of the falling charged dust shell. Then the related Vaidya AdS black brane is

$$
d s^{2}=\frac{1}{z^{2}}\left[-f(v, z) d v^{2}-2 d v d z+d x^{2}+d y^{2}\right],
$$

with $f(v, z)=1-\frac{1}{2} \beta^{2} z^{2}-M(v) z^{3}+Q(v)^{2} z^{4}$,

$$
\text { and } A_{v}=2 Q(v)(1-z) .
$$

Now, $v$ stands for the ingoing null trajectory, which coincides with the time coordinate $t$ on the conformal boundary. It is easy to check when $v \rightarrow+\infty$, the above formula reduces to the black brane solution (5), while in the limit $v \rightarrow-\infty$, it can recover the pure AdS spacetime when $\beta=0$.

Following the strategy of [51], we obtain the above solution (10)-(12) that corresponds to the external sources of current and energy-momentum tensor,

$$
\begin{gathered}
J_{(\mathrm{ext})}^{z}=2 \frac{d Q(v)}{d v}, \\
T_{v v}^{(\mathrm{ext})}=z^{2} \frac{d M(v)}{d v}-2 z^{3} Q(v) \frac{d Q(v)}{d v},
\end{gathered}
$$

respectively. It is noticed that in order to probe the timedependent optical conductivity without translation invariance, more external sources were considered to construct the solution in Vaidya setup of the EMA theory [42]. However, here in our solution, we consider the simple case that only the mass and charge depend on the time but the momentum relaxation coefficient $\beta$ does not vary with time. This is reasonable because as addressed in [43] for fivedimensional case that $M$ and $Q$ are integration constants when solving the differential equations while $\beta$ is fixed when sourcing the scalars.

\section{HOLOGRAPHIC SETUP OF HEE AND HC FOR A STRIPE}

In this section, we shall address the holographic setup of HEE and HC under a thermal quench in the field theory with momentum relaxation, which is dual to the bulk with axions described in the last section. We first consider the subregion with a straight strip geometry described by $\mathcal{A} \equiv\left\{x \in\left(-\frac{l}{2}, \frac{l}{2}\right), y \in\left(-\frac{L}{2}, \frac{L}{2}\right)\right\}$; see Fig. 1 where we will set the width $l$ to be finite and the length of the region $\mathcal{A}$ to be $L \rightarrow \infty$. As proposed in [5] that in the dynamical spacetime, HEE for a subregion $\mathcal{A}$ on the boundary is captured by the HRT bulk surface $\gamma_{\mathcal{A}}$, while the corresponding $\mathrm{HC}$ is proportional to the volume of a codimensionone hypersurface $\Gamma_{\mathcal{A}}$ with the boundaries $\mathcal{A}$ and $\gamma_{\mathcal{A}}$. Thus, we will follow the steps in [28] to analytically deduce the expressions of HEE via the minimal surface and $\mathrm{HC}$ via $\mathrm{CV}$ conjecture of the boundary theory. It is noticed that other probes such as Wilson loop and HEE in static case [52] and during thermal quench [53] in this theory with momentum relaxation have been investigated.

Due to the symmetry of the system, one can parametrize the corresponding extremal surface $\gamma_{\mathcal{A}}$ in the bulk as

$$
\begin{aligned}
v & =v(x), \quad z=z(x), \quad z( \pm l / 2)=\epsilon, \\
v( \pm l / 2) & =t-\epsilon,
\end{aligned}
$$

where $\epsilon$ is the UV cutoff. Then the induced metric on the surface is

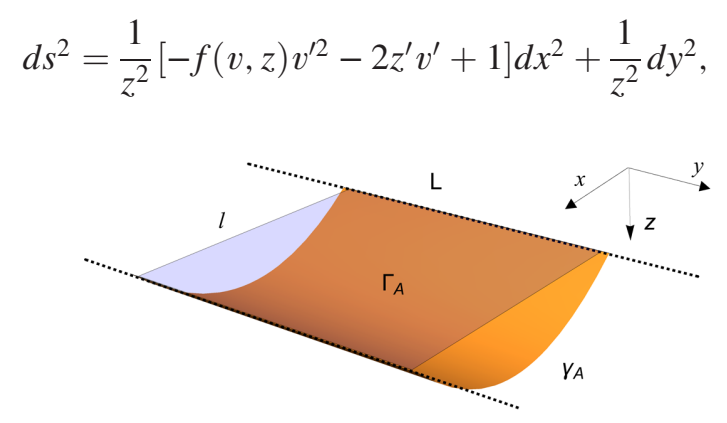

FIG. 1. Geometrical description of the subregion $\mathcal{A}$ (the light blue area) with width $l$ and length $L$. The yellow area indicates the HRT bulk surface $\gamma_{\mathcal{A}}$ while the hypersurface $\Gamma_{\mathcal{A}}$ is the bulk area with boundaries $\mathcal{A}$ and $\gamma_{\mathcal{A}}$. 
where the prime means taking the derivative to $x$. Then the area of the extremal surface is calculated as

$$
\operatorname{Area}\left(\gamma_{\mathcal{A}}\right)=L \int_{-l / 2}^{l / 2} \frac{\sqrt{1-f(v, z) v^{\prime 2}-2 z^{\prime} v^{\prime}}}{z^{2}} d x .
$$

To obtain the HEE, one has to minimize the above area. The trick is to treat the above function as an action with $x$ instead of "time," and so the related Lagrangian and Hamiltonian density are

$$
\begin{gathered}
\mathcal{L}_{S}=\frac{\sqrt{1-f(v, z) v^{\prime 2}-2 z^{\prime} v^{\prime}}}{z^{2}}, \\
\mathcal{H}_{S}=\frac{1}{z^{2} \sqrt{1-f(v, z) v^{\prime 2}-2 z^{\prime} v^{\prime}}} .
\end{gathered}
$$

It is obvious that the Hamiltonian does not explicitly depend on the variable $x$, so it is conserved. Besides, the symmetry of the surface implies a turning point $\left(z_{*}, v_{*}\right)$ at $x=0$ on the extremal surface $\gamma_{\mathcal{A}}$ (see Fig. 1 for a fixed $v$ ). Then, we can set

$v^{\prime}(0)=z^{\prime}(0)=0, \quad z(0)=z_{*}, \quad v(0)=v_{*}$.

Subsequently, the conserved Hamiltonian give us a condition,

$$
1-f(v, z) v^{2}-2 z^{\prime} v^{\prime}=\frac{z_{*}^{4}}{z^{4}}
$$

One can take derivative of the Lagrangian (18) with respect to $x$. Combining the derivative equation and the equations of motion for $z(x)$ and for $v(x)$, respectively, we get a group of partial differential equations,

$0=-4+2 z v^{\prime \prime}+v^{\prime}\left[4 f(v, z) v^{\prime}+8 z^{\prime}-z v^{\prime} \partial_{z} f(v, z)\right]$,

$$
\begin{aligned}
0= & 4 f(v, z)^{2} v^{2}+f(v, z)\left[-4+8 v^{\prime} z^{\prime}-z v^{2} \partial_{z} f(v, z)\right] \\
& -z\left[2 z^{\prime \prime}+v^{\prime}\left(2 z^{\prime} \partial_{z} f(v, z)+v^{\prime} \partial_{v} f(v, z)\right)\right] .
\end{aligned}
$$

By solving the above equations using the boundary conditions (20), one can extract the solutions of $v=$ $\tilde{v}(x), z=\tilde{z}(x)$ for the extremal surface $\gamma_{\mathcal{A}}$. Then the area of the extremal surface $\gamma_{\mathcal{A}}$ is simplified as

$$
\operatorname{Area}\left(\gamma_{\mathcal{A}}\right)=2 L \int_{0}^{l / 2} \frac{z_{*}^{2}}{\tilde{z}(x)^{4}} d x
$$

which gives the HEE of the subregion on the boundary. It is noted that the surface does not live on a constant time slice for the general $f(v, z)$, and both $z_{*}$ and $\tilde{z}(x)$ are time dependent.
Using the same profile as HEE, we then further derive the general expression of $\mathrm{HC}$ by evaluating the dual volume in the bulk of the background (5), i.e., we should evaluate the volume with the codimension-one extremal surface $\Gamma_{\mathcal{A}}$ which is bounded by the surface $\gamma_{\mathcal{A}}$. As discussed in [28], depending on the parametrization, there are two schemes to describe $\Gamma_{\mathcal{A}}$ which is enclosed by $v=\tilde{v}(x), z=\tilde{z}(x)$.

One scheme is to parametrize $\Gamma_{\mathcal{A}}$ via $z=z(v)$. Thus, the induced metric on $\Gamma_{\mathcal{A}}$ is

$$
d s^{2}=\frac{1}{z^{2}}\left[-\left(f(v, z)+2 \frac{\partial z}{\partial v}\right) d v^{2}+d x^{2}+d y^{2}\right]
$$

Consequently, the volume can be evaluated as

$V\left(\Gamma_{\mathcal{A}}\right)=2 L \int_{v_{*}}^{\tilde{v}(l / 2)} d v \int_{0}^{\tilde{x}(v)} \frac{d x}{z^{3}}\left[-f(v, z)-2 \frac{\partial z}{\partial v}\right]^{1 / 2}$,

where $\tilde{x}(v)$ is one coordinate in the codimension-two extremal surface $\gamma_{\mathcal{A}}$. We treat the above integral function as a Lagrangian, and then the corresponding equation of motion is

$$
\begin{aligned}
0= & 6 f(v, z)^{2}+12 z^{\prime}(v)^{2}-3 z(v) z^{\prime}(v) \partial_{z} f(v, z) \\
& +f(v, z)\left(18 z^{\prime}(v)-z(v) \partial_{z} f(v, z)\right) \\
& -z(v)\left(2 z^{\prime \prime}(v)+\partial_{v} f(v, z)\right) .
\end{aligned}
$$

There are two possible ways to get the solution to the above equation. One is to solve it with the use of the boundary conditions determined by the codimension-two surface $\gamma_{\mathcal{A}}=(\tilde{v}(x), \tilde{z}(x))$ and $\mathcal{A}$. The other is to figure out the solution by seeking $\tilde{z}(\tilde{v})$ on the boundary $\gamma_{\mathcal{A}}$. Subsequently, the volume (30) can be further written as

$V\left(\Gamma_{\mathcal{A}}\right)=2 L \int_{v_{*}}^{\tilde{v}(l / 2)} \frac{d v}{z(v)^{3}}\left[-f(v, z(v))-2 \frac{\partial z}{\partial v}\right]^{1 / 2} \tilde{x}(v)$.

The other scheme is to parametrize $\Gamma_{\mathcal{A}}$ via $v=v(z)$, in which the induced metric on the codimension-one surface is

$d s^{2}=\frac{1}{z^{2}}\left[-\left(f(v, z) \frac{\partial v}{\partial z}+2\right) \frac{\partial v}{\partial z} d z^{2}+d x^{2}+d y^{2}\right]$.

Subsequently, following the same step in the former scheme, the on-shell volume is reduced as 


$$
V\left(\Gamma_{\mathcal{A}}\right)=2 L \int_{0}^{z_{\star}} \frac{d z}{z^{3}}\left[-f(v(z), z)\left(\frac{\partial v}{\partial z}\right)^{2}-2 \frac{\partial v}{\partial z}\right]^{1 / 2} \tilde{x}(z) .
$$

It was pointed out in [28] that there are cases that $\tilde{v}(z)$ and $\tilde{x}(z)$ are multivalued functions of $z$ (this can also be seen in our late study), so the integral (30) is not always well defined in the whole process of evolution. While $\tilde{z}(\tilde{v})$ is a single valued function of $\tilde{v}$ all the time, so the integral (28) is well defined during the evolution. Moreover, the expression (30) is more intuitive than (28) in the static background, and their outcome is the same when $\tilde{v}(z)$ is singly valued function of $z$. Consequently, we will employ the integral (28) to compute the HC in the next discussion.

So far, we have obtained the explicit expressions of HEE (24) and HC (28) of the boundary theory dual to the background (2) under a thermal quench. In the following section, we will show our numerical results presenting the evolving behaviors of HEE and HC.

\section{NUMERICAL RESULTS}

To numerically study the evolution of HEE and HC, we have to solve the equations of motion (22) and (23) with the boundary conditions,

$$
\begin{aligned}
v^{\prime}(0) & =z^{\prime}(0)=0, \quad z(0)=z_{*}, \quad v(0)=v_{*}, \\
z(l / 2) & =\epsilon, \quad v(l / 2)=t-\epsilon .
\end{aligned}
$$

Conventionally, we are only interested in the finite physical quantities while both $\mathrm{HEE}$ and $\mathrm{HC}$ evaluated by above conditions are divergent if $\epsilon \rightarrow 0$. Therefore, we could define the following relative renormalized finite terms:

$$
\begin{gathered}
S=\frac{\operatorname{Area}\left(\gamma_{\mathcal{A}}\right)-\operatorname{Area}_{\mathrm{AdS}}\left(\gamma_{\mathcal{A}}\right)}{2 L}, \\
C=\frac{V\left(\Gamma_{\mathcal{A}}\right)-V_{\mathrm{AdS}}\left(\Gamma_{\mathcal{A}}\right)}{2 L},
\end{gathered}
$$

where $\operatorname{Area}\left(\gamma_{\mathcal{A}}\right)$ and $V\left(\Gamma_{\mathcal{A}}\right)$ are defined in Eqs. (24) and (28), respectively. The quantities with subscript $A d S$ correspond to the vacuum part dual to the AdS geometry with vanishing mass and charge, in which we have to find the minimal surface $\gamma_{\mathcal{A}}$ and the related volume of $\Gamma_{\mathcal{A}}$, separately. Usually, they can be integrated out as $\operatorname{Area}_{\mathrm{AdS}}\left(\gamma_{\mathcal{A}}\right)=\frac{2 L}{\epsilon}+\frac{L}{2 z_{*}} \frac{\sqrt{\pi} \Gamma\left(-\frac{1}{4}\right)}{\Gamma\left(\frac{1}{4}\right)}$ and $\mathrm{V}_{\mathrm{AdS}}\left(\Gamma_{\mathcal{A}}\right)=\frac{L l}{2 \epsilon^{2}}+\frac{\sqrt{\pi} L}{z_{*}}\left(\frac{2 \Gamma\left(\frac{3}{4}\right)}{\Gamma\left(\frac{1}{4}\right)}-\frac{3 \Gamma\left(\frac{1}{4}\right)}{4 \Gamma\left(\frac{3}{4}\right)}\right)$, respectively.

Next, we present the numerical results for the evolutions of these two quantities with quench. For the sake of the numerical precision, we will set the UV cutoff $\epsilon=0.05$, the thickness of the shell $v_{0}=0.01$, and the mass parameter $m=1$ in the calculation.

\section{A. Evolution of HEE and $\mathrm{HC}$ in neutral case}

To see the role of momentum dissipation plays in the evolution of HEE and HC, we first consider the neutral case, i.e., $q=0$ so we can focus on the effect of $\beta$. In addition, we fix the strip width $l=2$ as the first step.

Before presenting the main results of HEE and HC, we first show the evolution of the HRT surface $\gamma_{\mathcal{A}}$ for $\beta=5$ to give an intuitive understanding of the evolution. The left panel in Fig. 2 exhibits the evolution in $(x, v, z)$ space, in which $\gamma_{\mathcal{A}}$ evolves from left to right. In the middle panel of Fig. 2, the corresponding projection in $(x, z)$ plan is shown, in which the evolution is from top to bottom. It is obvious that $\gamma_{\mathcal{A}}$ evolves smoothly from the initial state to the final state. After obtaining the HRT surface $\gamma_{\mathcal{A}}$, we can then work out the codimension-one surface $\Gamma_{\mathcal{A}}$, which characterizes the subregion complexity bounded by the HRT surface $\gamma_{\mathcal{A}}$ as well as subregion $\mathcal{A}$, which is exhibited in the right of Fig. 2. The process is similar with the case in the Einstein gravity in [28].

The evolution of HEE and HC affected by $\beta$ is shown in Fig. 3. Several properties can be read off from the figures. First, the left panel shows the evolution of the turning point $z_{*}$ over the horizon radius $z_{h}$. We see that at the beginning of evolution, since $z_{h}$ decreases sharply as the horizon radius increases suddenly under the quench function (8),
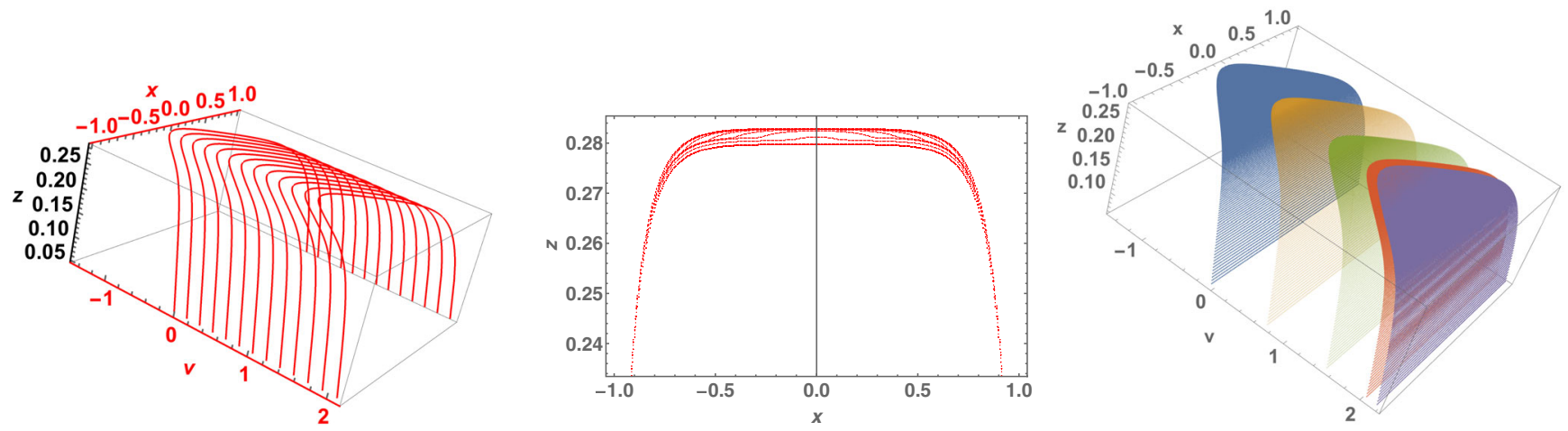

FIG. 2. Left: the evolution of HRT surface in $(x, v, z)$ space. Middle: the projection of HRT surface in $(x, z)$ planer. Right: the volume of $\Gamma_{\mathcal{A}}$. Here we have set $\beta=5$ and $l=2$. 

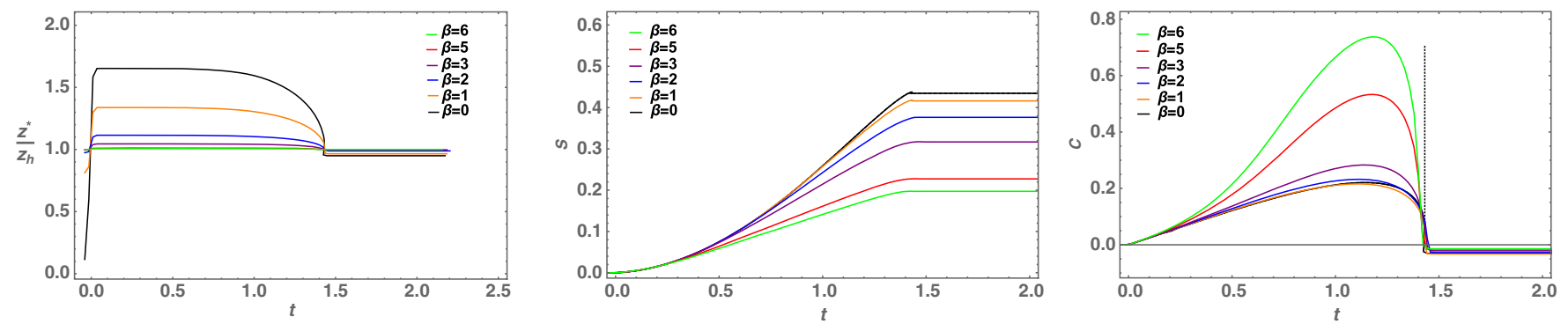

FIG. 3. Left: the evolution of $z_{*} / z_{h}$ for different $\beta$. Middle: the evolution of HEE for different $\beta$. Right: the relation between $\mathrm{HC}$ and $\beta$. Here we have set $l=2$.
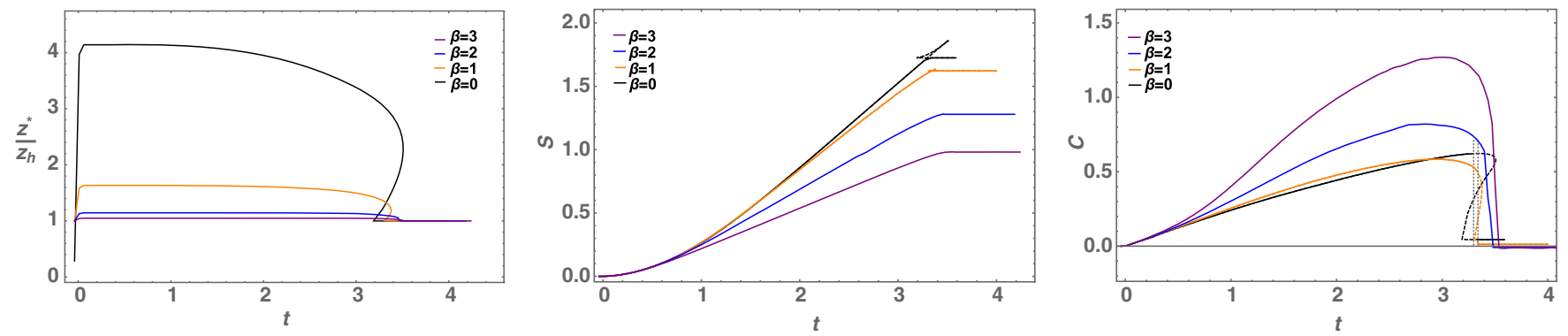

FIG. 4. Left: the evolution of $z_{*} / z_{h}$ for different $\beta$. Middle: the evolution of HEE for different $\beta$. Right: the relation between the HC and $\beta$. Here we have set $l=5$.

the ratio $z_{*} / z_{h}$ increases sharply to be larger than 1 , meaning the HRT surface is outside the horizon. As time passes, the ratio drops and approaches to 1 which implies that the HRT surface is always inside the horizon as the system becomes saturate. The corresponding stable HEE in the middle panel for larger $\beta$ is smaller. In the contrary, the right panel shows that $\beta$ slightly affects the stable value of $\mathrm{HC}$ while its peak is explicitly higher. We then study the effect of $\beta$ on $\mathrm{HEE}$ and $\mathrm{HC}$ with wide subregion, i.e., $l=5$. The results are plotted in Fig. 4.

For $\beta=0$, we reproduced the result of four-dimensional Schwashiz black hole [28], which is denoted by the black lines in each plot. For different $\beta$, HEE has swallow-tail behaviors and $\mathrm{HC}$ has the multivalued regions, but only solid lines describe the physical procedure. This phenomenon can be explained by the evolution of $\gamma_{\mathcal{A}}$ shown in Fig. 5, which is no longer a continuous function and different from the case of the small width strips. The discontinuous evolution, i.e., the dropping behavior is related with the jump in the minimal area surface from phenomenon perspective.

For nonvanishing $\beta$, we find that the effect of momentum relaxation for wider strip is more explicit than that for narrow strip by comparing Figs. 3 and 4. More interesting properties can be observed, which are summarized as follows:

(i) The turning point $z_{*} / z_{h}$, relative $\mathrm{HEE}$ and $\mathrm{HC}$ all tend to be constants as time evolves Specially, as $\beta$ increases, all the relative renormalized stable values become smaller. But it should be noticed that after saturation, $z_{*} / z_{h} \sim 1$, so one could expect $\gamma_{\mathcal{A}}$ wraps the horizon completely and the stable value of
HEE is equal to the thermal entropy (thermal entropy density (4) times the volume) [54]. In our model, the thermal entropy density increases as $\beta$ increases because larger $\beta$ corresponds to lower $z_{h}$ (i.e., larger horizon radius $r_{h}$ ), so we could compute the stable HEE using basic thermodynamics and the stable value of HEE should become larger if $\beta$ is large. ${ }^{1}$ However, this phenomenon is not in conflict with our numerical results because here we describe the relative renormalized HEE [see Eq. (32)].

(ii) A novel feature is that as $\beta$ increases to be a certain value, the swallow-tail behavior in HEE and the multivalued region in $\mathrm{HC}$ disappear as shown in Fig. 4. In other words, the evolution of $\gamma_{\mathcal{A}}$ recovers to continue. It implies that comparing to the case in Einstein gravity, in this model with axion field, the system with wide strip can emerge discontinue evolution of $\gamma_{\mathcal{A}}$. We note that we do not observe the discontinue recovers as we further increase $\beta$ as we can.

(iii) Figure 3 shows that for $l=2$, the larger $\beta$ promotes the system to become equilibrium. That is to say, larger $\beta$ needs less time to reach the stable state in thermalization. While for $l=5$ in Fig. 4 , larger $\beta$

\footnotetext{
${ }^{1}$ Via subtracting the divergent term $2 L / \epsilon$, we have computed the original HEE, (24), for the static background (2) which is the stable state under thermalization. We obtain that the value of $\mathrm{HEE}$ indeed increases as $\beta$ which is consistent with that for the thermal entropy density.
} 

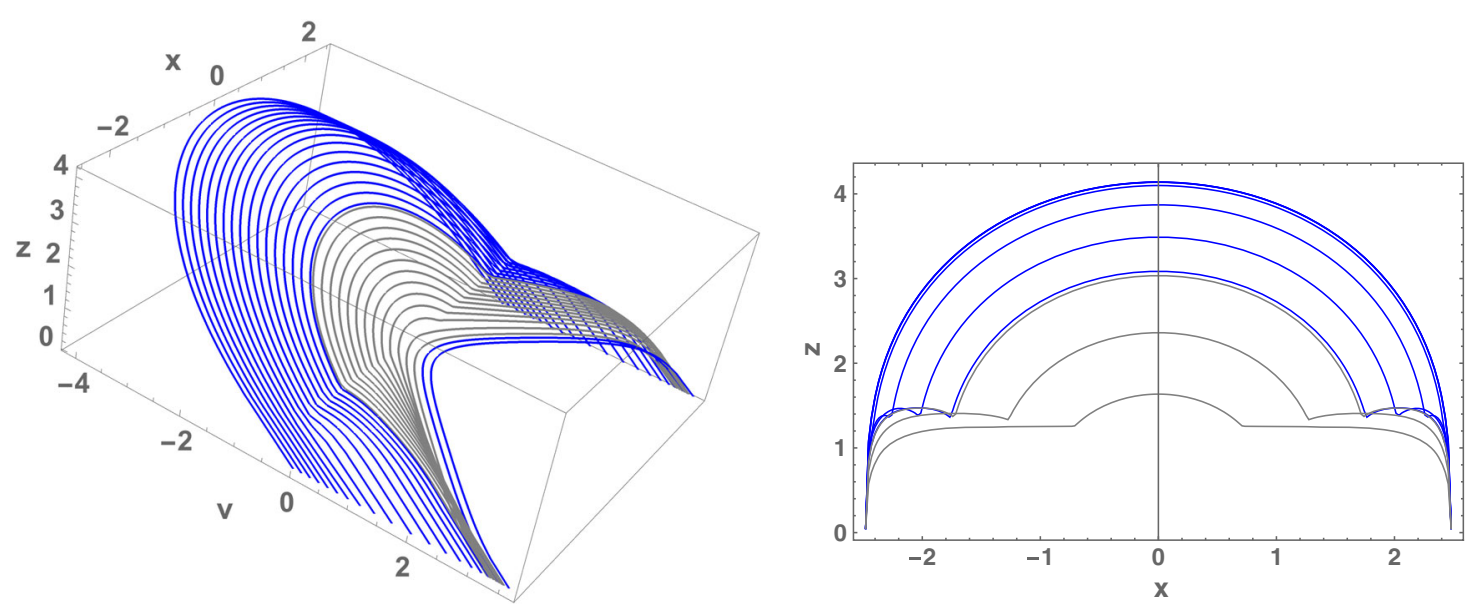

FIG. 5. Left: the evolution of HRT surface in $(x, v, z)$ space. Right: the projection of HRT surface in $(x, z)$ planer. Here we have set $\beta=0$ and $l=5$.
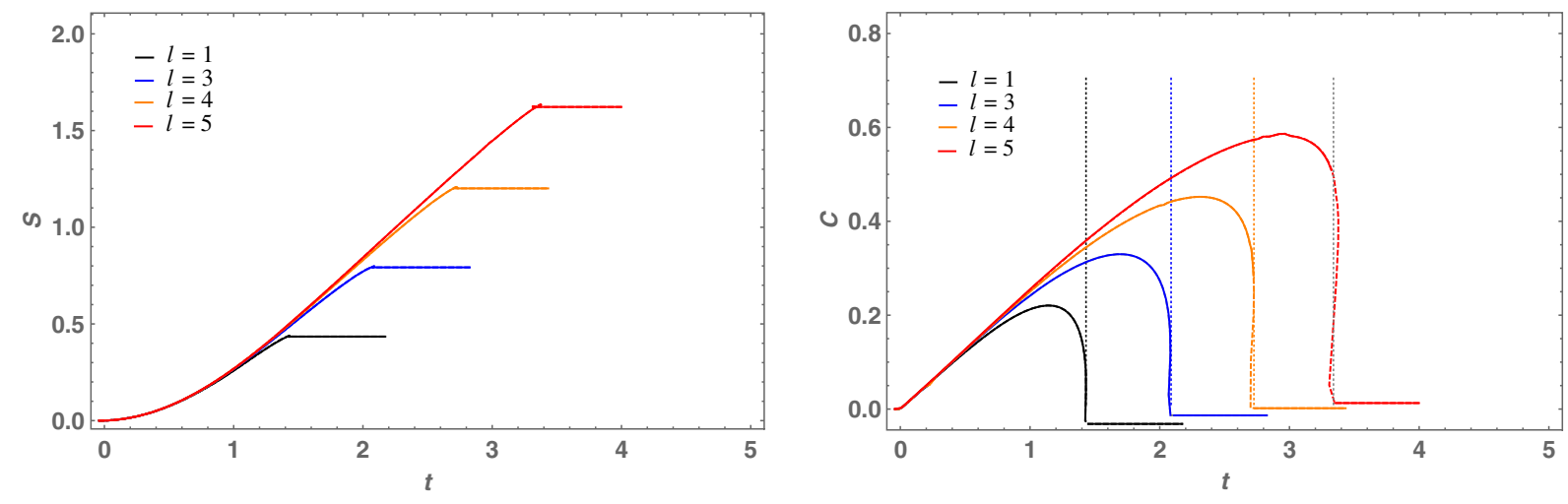

FIG. 6. The evolution of HEE and HC with samples of stripe width for $\beta=1$.

corresponds to longer time to become stable. Moreover, larger $\beta$ in this case is related with lower stable value of both HEE and $\mathrm{HC}$, which is also different from that $\beta$ suppresses the stable HEE but promotes $\mathrm{HC}$ for $l=2$. This property suggests that if we fix $q$ in advance, the time that HEE or HC needs to reach the stable values may not monotonically depend on $\beta$ for fixed $l$, and vice versa.

(iv) The systems with large $l$ need more time to be stable than those with small $l$. This rule can also be explicitly extracted from Fig. 6 where we present the evolution of HEE and HC with different $l$ for fixed $\beta=1$. This feature was also observed in Einstein case [28] and Einstein-Born-Infeld case [31] which can be directly explained via the picture of entanglement tsunami proposed in $[55,56]$.

Note that since the numeric instability for large $\beta$ and $l$, we only exhibit the results for $\beta \leq 3$ in Fig. 4. But we expect that it is enough to show the universal characteristic for large $\beta$. In future, we would also like to improve our algorithm to work out the results for large $\beta$ such that we can confirm the universal characteristic for large $\beta$. It is also interesting to analytically study HEE and $\mathrm{HC}$ in the large $\beta$ limit, which shall help to confirm the universal characteristic.

It is interesting and significant to understand the above results from analytical perspective. It was proposed in $[55,56]$ that many properties of the time evolution of HEE after a thermal quench can be analytically calculated with the picture of entanglement tsunami, and later the authors of [30] apply the same strategy to study the time evolution of HC. As they addressed that their calculations were based on that the metric function satisfies some properties, one of which is the asymptotical behavior $f(z) \sim 1-M z^{d}+\cdots$ as $z \rightarrow 0$. However, in our study, the asymptotical behavior is $f(z) \sim 1-\frac{1}{2} \beta^{2} z^{d-1}+M z^{d}+\cdots$, and the subleading term is not $z^{d}$ order but $z^{d-1}$ order with nonvanishing $\beta$. Thus, the calculation and conclusion of $[30,55,56]$ could not generalize to our case. We hope to develop the analytical study in our model in the near future.

\section{B. Evolution of HEE and $\mathrm{HC}$ in charged case}

In this section, we shall turn on the charge in this model and study the joined effect of $\beta$ and $q$ on the evolution of HEE and HC. 

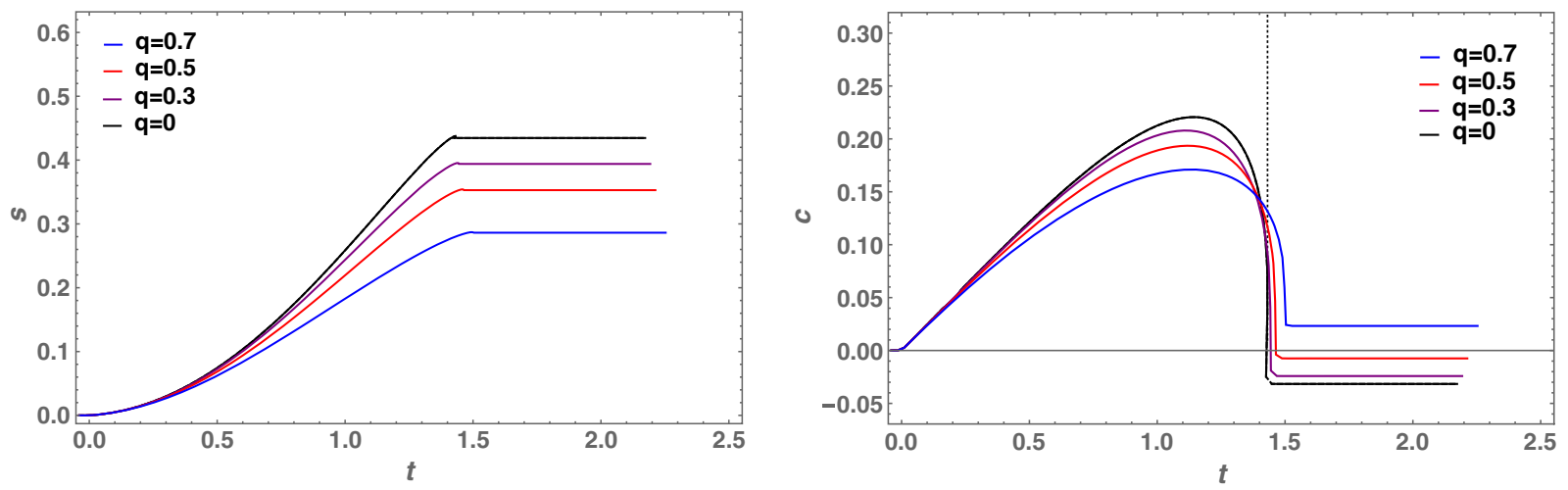

FIG. 7. The evolution of HEE (left panel) and HC (right panel) for different $q$ with $l=2, \beta=1$.
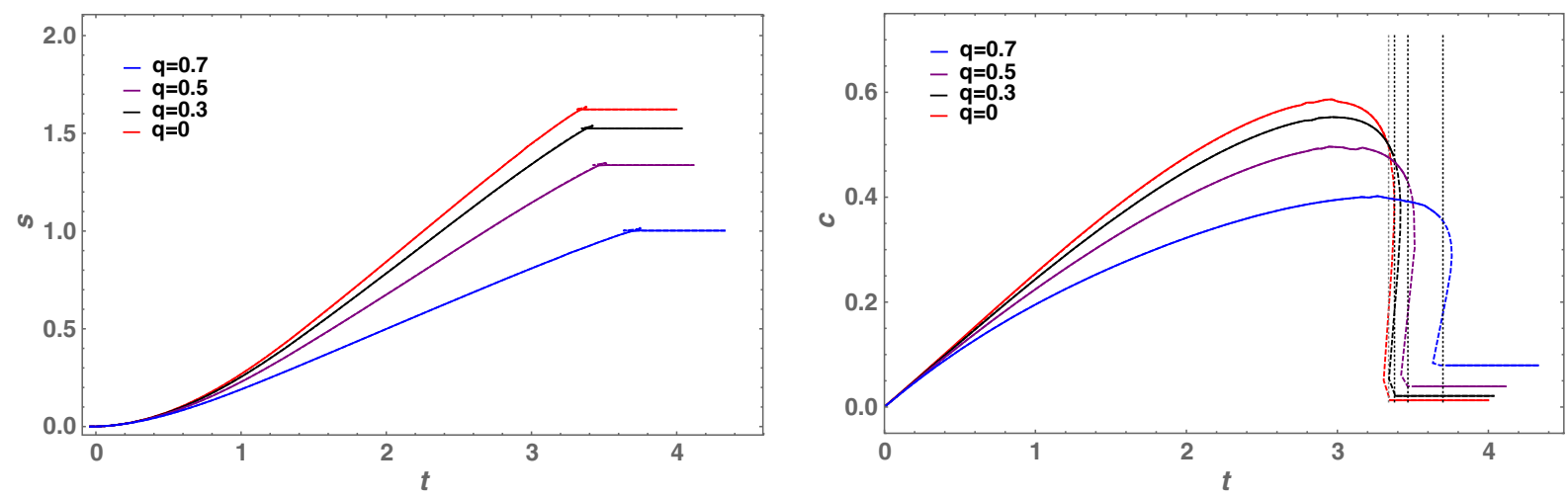

FIG. 8. The evolution of HEE (left panel) and HC (right panel) for different $q$ with $l=5, \beta=1$. The shallow-tail behaviors and multivalued regions are clearer than $l=2$ case.

It is noticed that the evolution of HEE and HC affected by the charge in four-dimensional Einstein theory and Einstein-Born-Infeld theory has been studied in [31]. We first repeat their results and then turn on $\beta=1$ to see the effect of charge in the model with momentum relaxation. Our results are shown in Fig. 7 for a small width $(l=2)$ and in Fig. 8 for a large width $(l=5)$. From the figures, we see that the charged black holes need longer time to become stable, and larger charge corresponds to smaller stable HEE but bigger stable HC. This phenomenon is similar as the existed observes in [31].

It is worthwhile to point out that for some parameters in our study, the final saturated HC could be almost zero and even negative, which seems to be universal behavior in the study of quench process with different models $[28,31,32,34,57]$. However, this is in contrast to
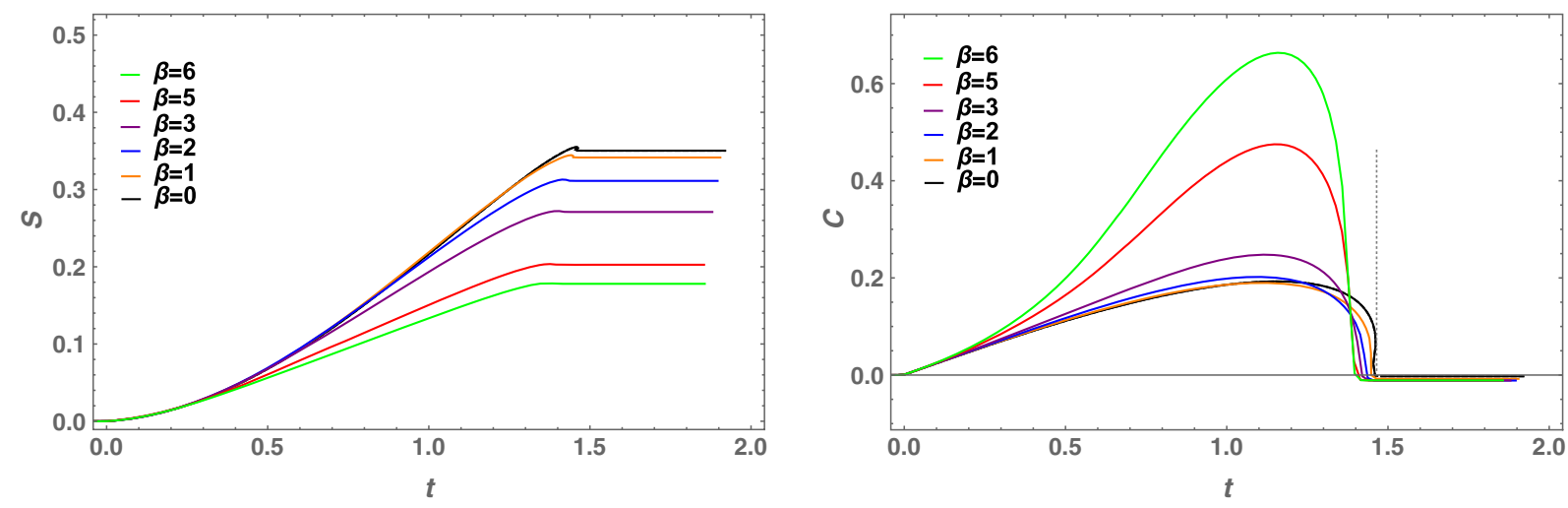

FIG. 9. Left: the evolution of HEE for different $\beta$. Right: the evolution of HC for different $\beta$. Here we have set $l=2, q=0.5$. 

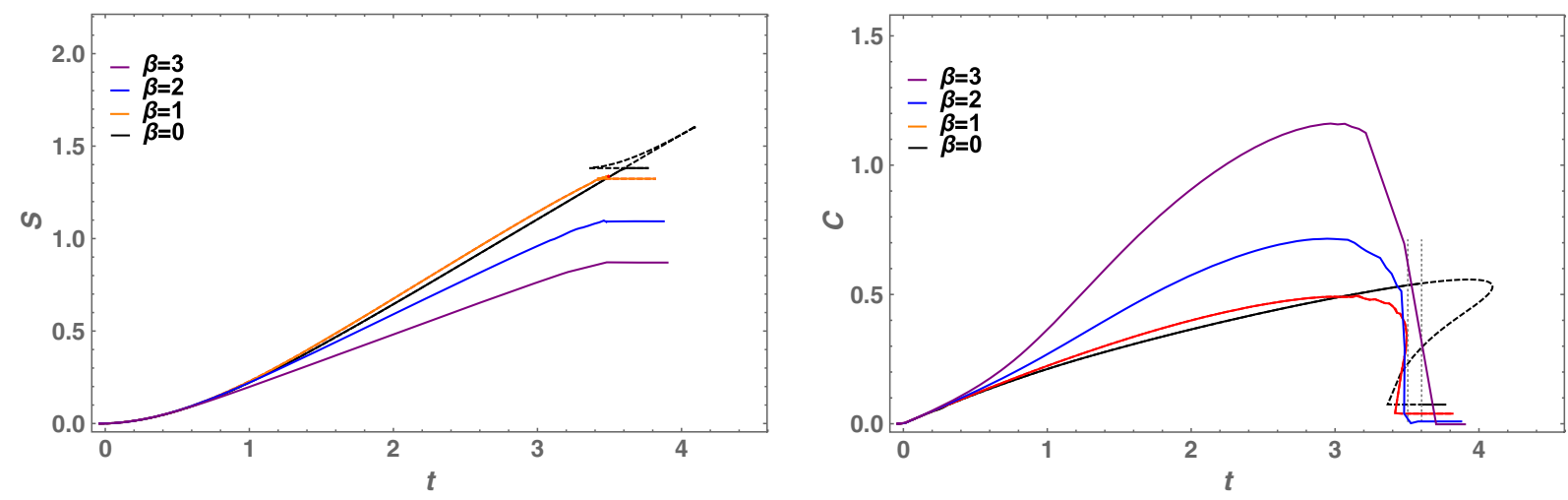

FIG. 10. Left: the evolution of HEE for different $\beta$. Right: the evolution of HC for different $\beta$. Here we have set $l=5, q=0.5$. Clearly, larger $\beta$ restrains the swallow tails and multivalued regions.

the behavior that the complexity is always a monotonically increasing function of time found in [58]. To understand this behavior could intrigue open questions: one is whether the normalized subregion complexity described via holography is dual to the complexity of formation of a state, which measures how difficult is to map a given reference state into a desired state. The other is what is the reference state in the holographic study. Since the aforementioned questions are still open, so the negative equilibrium $\mathrm{HC}$, which means that the stable value of the complexity is smaller than its initial value, could be acceptable but called for further understanding.

Comparing Figs. 7 and 8, it is obvious that, again, larger size of width brings in swallow tails in HEE and multivalues in $\mathrm{HC}$ due to the discontinue behavior of minimum area. Moreover, Fig. 8 shows that larger $q$ makes the discontinue behavior more evident, which is similar to that found in [31,32].

In the charged case with $q=0.5$, we show the evolution of HEE and HC for different $\beta$ in Fig. $9(l=2)$ and Fig. 10 $(l=5)$. For $l=2$, the evolution is still continue as shown in Fig. 9. While in Fig. 10, they are discontinue and $\beta$ also suppresses the effect of $l$, so that the swallow-tail and multivalues behavior disappear as we increase $\beta$, which is similar to what we observe in neutral case.

\section{CONCLUSION AND DISCUSSION}

In this paper, we studied the evolution of HEE and HC under a thermal quench in EMA theory. In this theory, the black brane solution is homogeneous but the dual boundary theory has momentum dispersion because the spacialdependent axion fields provide the source to break the translation symmetry. We mainly investigated the effects of the momentum relaxation and the charge of the black brane on the evolution of HEE and HC.

In neutral case, when $l$ is tuned large, the evolutions of HEE and HC behave from a continuous function into a discontinuous one. For the continuous case, HEE increases at the first stage and then it arrives at a stable final region, while $\mathrm{HC}$ grows until it arrives at a maximum point, and then after that it quickly drops to become a stable final stage. For the discontinuous case, a swallow tail appears in the evolution of HEE while correspondingly a multivalued behavior can be seen in HC. This picture is the same as that found in [28,31]. However, as the momentum relaxation increases, the swallow-tail behavior in HEE and the multivalued region in $\mathrm{HC}$ disappear, i.e., the continuous evolutions will be recovered. This denotes that in this model, the system with larger width would lead to discontinuous evolution of $\gamma_{\mathcal{A}}$ in contrast to that in Einstein theory. Moreover, large $\beta$ corresponds to smaller stable value of both HEE and $\mathrm{HC}$, while the time that HEE or HC needs to reach the stable values is not monotonically depended on $\beta$ for fixed $l$, and vice versa. It would be interesting to understand our results from analytical approach.

We found that the charge would make the discontinue behavior in HEE and $\mathrm{HC}$ more explicit at large $l$. That is to say, for bigger charge, the system with narrower size could emerge discontinuity in the evolution. This phenomenon has also been observed in [31,32]. Similarly, to as occurred in neutral case, when we increase the momentum relaxation, the swallow tail in HEE and the multivalued region in $\mathrm{HC}$ become weaken and finally the continuous evolutions would be recovered.

In all of the evolutions we plotted, it is obvious that at the early stage the behavior of evolution is almost the same, i.e., they almost do not depend on the parameters including the size the strip. This is because the growth of complexity stems from the local operator excitations. The possible bound of HEE growth rate for various stages of evolution has been discussed in [56], while the Lloyd bound for HC growth rate $d C(t) / d t \leq 2 M / \pi$, where $M$ is total mass of the system at any time $t$, has been addressed in [8]. Thus, it would be very interesting to carefully analyze the growth rate in various stages of the evolution of HEE and HC in our model and compare them with the related bounds. This work is under progress. 
Besides HEE and HC, our studies can be extended into the related quantities we mentioned in the introduction, i.e., EoP, CoP, and logarithmic negativity and their evolution under the thermal quench using similar methods. EoP of mixed state in the dual theory of this model with momentum relaxation has been studied in [59] very recently. The evolutions of EoP in this setup of Einstein gravity can be seen in [60], and it is natural to ask how its evolution will be affected when the momentum dispersion is involved in the system.

\section{ACKNOWLEDGMENTS}

We appreciate Cheng-Yong Zhang for helpful discussions. This work is supported by the Natural Science Foundation of China under Grants No. 11705161, No. 11775036, and No. 11847313, and Fok Ying Tung Education Foundation under Grant No. 171006. X.-M. K. is also supported by the Natural Science Foundation of Jiangsu Province under Grant No. BK20170481. J.-P. W. is also supported by Top Talent Support Program from Yangzhou University.
[1] J. M. Maldacena, The large N limit of superconformal field theories and supergravity, Int. J. Theor. Phys. 38, 1113 (1999) [Adv. Theor. Math. Phys. 2, 231 (1998)].

[2] S. S. Gubser, I. R. Klebanov, and A. M. Polyakov, Gauge theory correlators from noncritical string theory, Phys. Lett. B 428, 105 (1998).

[3] E. Witten, Anti-de Sitter space and holography, Adv. Theor. Math. Phys. 2, 253 (1998).

[4] T. Takayanagi, Entanglement entropy from a holographic viewpoint, Classical Quantum Gravity 29, 153001 (2012).

[5] V.E. Hubeny, M. Rangamani, and T. Takayanagi, A covariant holographic entanglement entropy proposal, J. High Energy Phys. 07 (2007) 062.

[6] D. Stanford and L. Susskind, Complexity and shock wave geometries, Phys. Rev. D 90, 126007 (2014).

[7] L. Susskind and Y. Zhao, Switchbacks and the bridge to nowhere, arXiv:1408.2823.

[8] A. R. Brown, D. A. Roberts, L. Susskind, B. Swingle, and Y. Zhao, Holographic Complexity Equals Bulk Action?, Phys. Rev. Lett. 116, 191301 (2016).

[9] A. R. Brown, D. A. Roberts, L. Susskind, B. Swingle, and Y. Zhao, Complexity, action, and black holes, Phys. Rev. D 93, 086006 (2016).

[10] A. Bhattacharya, K. T. Grosvenor, and S. Roy, Entanglement entropy and subregion complexity in thermal perturbations around pure-AdS spacetime, Phys. Rev. D 100, 126004 (2019).

[11] P. Braccia, A. L. Cotrone, and E. Tonni, Complexity in the presence of a boundary, J. High Energy Phys. 02 (2020) 051.

[12] B. M. Terhal, M. Horodecki, D. W. Leung, and D. P. DiVincenzo, The entanglement of purification, J. Math. Phys. (N.Y.) 43, 4286 (2002).

[13] T. Takayanagi and K. Umemoto, Entanglement of purification through holographic duality, Nat. Phys. 14, 573 (2018).

[14] P. Nguyen, T. Devakul, M. G. Halbasch, M. P. Zaletel, and B. Swingle, Entanglement of purification: From spin chains to holography, J. High Energy Phys. 01 (2018) 098.

[15] K. Umemoto and Y. Zhou, Entanglement of purification for multipartite states and its holographic dual, J. High Energy Phys. 10 (2018) 152.
[16] P. Liu, Y. Ling, C. Niu, and J. P. Wu, Entanglement of purification in holographic systems, J. High Energy Phys. 09 (2019) 071.

[17] M. Ghodrati, X. M. Kuang, B. Wang, C. Y. Zhang, and Y. T. Zhou, The connection between holographic entanglement and complexity of purification, J. High Energy Phys. 09 (2019) 009.

[18] J. Harper and M. Headrick, Bit threads and holographic entanglement of purification, J. High Energy Phys. 08 (2019) 101.

[19] N. Bao, A. Chatwin-Davies, J. Pollack, and G. N. Remmen, Towards a bit threads derivation of holographic entanglement of purification, J. High Energy Phys. 07 (2019) 152.

[20] D. H. Du, C. B. Chen, and F. W. Shu, Bit threads and holographic entanglement of purification, J. High Energy Phys. 08 (2019) 140.

[21] C. A. Agon, M. Headrick, and B. Swingle, Subsystem complexity and holography, J. High Energy Phys. 02 (2019) 145.

[22] M. B. Plenio, Logarithmic Negativity: A Full Entanglement Monotone That is Not Convex, Phys. Rev. Lett. 95, 119902 (2005).

[23] Y. Kusuki, J. Kudler-Flam, and S. Ryu, Derivation of holographic negativity in $\mathrm{AdS}_{3} / \mathrm{CFT}_{2}$, Phys. Rev. Lett. 123, 131603 (2019).

[24] J. Kudler-Flam and S. Ryu, Entanglement negativity and minimal entanglement wedge cross sections in holographic theories, Phys. Rev. D 99, 106014 (2019).

[25] L. Susskind, Computational complexity and black hole horizons, Fortschr. Phys. 64, 44 (2016).

[26] L. Susskind, Entanglement is not enough, Fortschr. Phys. 64, 49 (2016).

[27] J. Maldacena and L. Susskind, Cool horizons for entangled black holes, Fortschr. Phys. 61, 781 (2013).

[28] B. Chen, W. M. Li, R. Q. Yang, C. Y. Zhang, and S. J. Zhang, Holographic subregion complexity under a thermal quench, J. High Energy Phys. 07 (2018) 034.

[29] R. Auzzi, G. Nardelli, F. I. Schaposnik Massolo, G. Tallarita, and N. Zenoni, On volume subregion complexity in Vaidya spacetime, arXiv:1908.10832.

[30] Y. Ling, Y. Liu, C. Niu, Y. Xiao, and C. Y. Zhang, Holographic subregion complexity in general Vaidya geometry, J. High Energy Phys. 11 (2019) 039. 
[31] Y. Ling, Y. Liu, and C. Y. Zhang, Holographic subregion complexity in Einstein-Born-Infeld theory, Eur. Phys. J. C 79, 194 (2019).

[32] Y. T. Zhou, M. Ghodrati, X. M. Kuang, and J. P. Wu, Evolutions of entanglement and complexity after a thermal quench in massive gravity theory, Phys. Rev. D 100, 066003 (2019).

[33] R. Q. Yang and K. Y. Kim, Time evolution of the complexity in chaotic systems: Concrete examples, arXiv:1906.02052.

[34] S. J. Zhang, Subregion complexity in holographic thermalization with dS boundary, Eur. Phys. J. C 79, 715 (2019).

[35] S. Leichenauer and M. Moosa, Entanglement tsunami in (1 + 1)-dimensions, Phys. Rev. D 92, 126004 (2015).

[36] S. Leichenauer, M. Moosa, and M. Smolkin, Dynamics of the area law of entanglement entropy, J. High Energy Phys. 09 (2016) 035.

[37] Z. Y. Fan and M. Guo, Holographic complexity under a global quantum quench, Nucl. Phys. B950, 114818 (2020).

[38] J. Chu, R. Qi, and Y. Zhou, Generalizations of reflected entropy and the holographic dual, J. High Energy Phys. 03 (2020) 151.

[39] M. Moosa, Divergences in the rate of complexification, Phys. Rev. D 97, 106016 (2018).

[40] T. Andrade and B. Withers, A simple holographic model of momentum relaxation, J. High Energy Phys. 05 (2014) 101.

[41] C. de Rham, G. Gabadadze, and A. J. Tolley, Resummation of Massive Gravity, Phys. Rev. Lett. 106, 231101 (2011).

[42] A. Bagrov, B. Craps, F. Galli, V. Kernen, E. Keski-Vakkuri, and J. Zaanen, Holography and thermalization in optical pump-probe spectroscopy, Phys. Rev. D 97, 086005 (2018).

[43] J. Fernndez-Pends and K. Landsteiner, Out of equilibrium chiral magnetic effect and momentum relaxation in holography, Phys. Rev. D 100, 126024 (2019).

[44] V. Balasubramanian, A. Bernamonti, J. de Boer, B. Craps, L. Franti, F. Galli, E. Keski-Vakkuri, B. Müller, and A. Schäfer, Inhomogeneous Thermalization in Strongly Coupled Field Theories, Phys. Rev. Lett. 111, 231602 (2013).

[45] L. Alberte, M. Baggioli, A. Khmelnitsky, and O. Pujolas, Solid holography and massive gravity, J. High Energy Phys. 02 (2016) 114.

[46] L. Q. Fang and X. M. Kuang, Holographic heat engine with momentum relaxation, Sci. Chin. Phys. Mech. Astron. 61, 080421 (2018).

[47] A. Cisterna, S. Q. Hu, and X. M. Kuang, Joule-Thomson expansion in AdS black holes with momentum relaxation, Phys. Lett. B 797, 134883 (2019).
[48] S. Q. Hu and X. M. Kuang, Holographic heat engine in Horndeski model with the $k$-essence sector, Sci. Chin. Phys. Mech. Astron. 62, 60411 (2019).

[49] D. Galante and M. Schvellinger, Thermalization with a chemical potential from AdS spaces, J. High Energy Phys. 07 (2012) 096.

[50] V. Balasubramanian, A. Bernamonti, J. de Boer, N. Copland, B. Craps, E. Keski-Vakkuri, B. Müller, A. Schäfer, M. Shigemori, and W. Staessens, Holographic thermalization, Phys. Rev. D 84, 026010 (2011).

[51] E. Caceres and A. Kundu, Holographic thermalization with chemical potential, J. High Energy Phys. 09 (2012) 055.

[52] M. R. M. Mozaffar, A. Mollabashi, and F. Omidi, Non-local probes in holographic theories with momentum relaxation, J. High Energy Phys. 10 (2016) 135.

[53] Y.Z. Li and X. M. Kuang, Probes of holographic thermalization in a simple model with momentum relaxation, Nucl. Phys. B956, 115043 (2020).

[54] S. Ryu and T. Takayanagi, Holographic Derivation of Entanglement Entropy from AdS/CFT, Phys. Rev. Lett. 96, 181602 (2006).

[55] H. Liu and S. J. Suh, Entanglement Tsunami: Universal Scaling in Holographic Thermalization, Phys. Rev. Lett. 112, 011601 (2014).

[56] H. Liu and S. J. Suh, Entanglement growth during thermalization in holographic systems, Phys. Rev. D 89, 066012 (2014).

[57] D. S. Ageev, I. Y. Arefeva, A. A. Bagrov, and M. I. Katsnelson, Holographic local quench and effective complexity, J. High Energy Phys. 08 (2018) 071.

[58] S. Chapman, H. Marrochio, and R. C. Myers, Holographic complexity in Vaidya spacetimes. Part I, J. High Energy Phys. 06 (2018) 046; Holographic complexity in Vaidya spacetimes. Part II, J. High Energy Phys. 06 (2018) 114.

[59] Y. f. Huang, Z. j. Shi, C. Niu, C. y. Zhang, and P. Liu, Mixed state entanglement for holographic axion model, arXiv:1911 10977.

[60] R. Q. Yang, C. Y. Zhang, and W. M. Li, Holographic entanglement of purification for thermofield double states and thermal quench, J. High Energy Phys. 01 (2019) 114. 